\title{
Environmental modelling - a modern tool towards sustainability
}

\author{
Gelu Madear ${ }^{*}$, and Camelia Madear ${ }^{2}$ \\ ${ }^{1}$ Eco-ROCCA Engineering, 3, Mihai Viteazu Street, Petrosani, Romania \\ ${ }^{2}$ University of Petrosani, 20, University Street, Petrosani, Romania
}

\begin{abstract}
One way to solve environmental problems is through modelling. Humankind developed a series of models, from mental models, physical models to computer simulation models. Building a model assumes abstraction, simplifying the natural system by considering only the essential details and discarding irrelevant ones. Mapping the real worlds to the world of models is done by choosing an abstraction level and the corresponding modelling tool. The right abstraction level is paramount for any modelling project, depending on the real problem being analysed. In modern simulation modelling, there are three methods, each having a particular range of abstraction levels: system dynamics, discrete event (process-centric modelling) and agent-based models. Ecosystems and generally any environmental problems (real world) are complex dynamics that challenge our comprehension. Understanding the significant environmental challenges is vital to adopt adequate policies for a sustainable environment through modelling and simulation. Since our cognitive abilities are limited, we need a simulation of the environmental systems to see the dynamic patterns and how humans interact with the environment. Environmental modelling helps us understand complex systems by building mathematical models and running simulations using a high abstraction level. The system dynamics method of modelling and simulation is used to clarify the representation of the stocks and flows and the feedback process that control the flows and describe the dynamic behaviour (growth, decay, or oscillations) of complex systems over time. Modelling for prediction, understanding across time and spatial scales, and environmental systems disciplines is key for a sustainable future.
\end{abstract}

\section{Introduction}

Using modelling is the way forward to solve the real world's problems and allow ideas to be deeply investigated. The right solution must be found, being aware that we cannot experiment by destroying, changing, and guessing. In most cases, apart from being dangerous or expensive, it is impossible.

\footnotetext{
${ }^{*}$ Corresponding author: gelu.madear@eco-rocca.ro
} 
We understand that a model is an abstraction that allows people to focus on a complex problem's fundamental by keeping out non-essential details. There is a specific limit to how much a person can understand; therefore, building models help in various activities.

Leaving the real world and go up to the world of models implies assuming abstraction, exploring, understanding the structure and behaviour of the original system, testing how the system will behave under various conditions, playing, comparing different scenarios, and optimising.

Finally, when we manage to figure out the solution we were looking for, we map that solution back to the real world. The main idea of modelling is to find a way from the problem to its solution in a risk-free environment where we can make errors, fix them, go back in time, and start over.

Models are less complicated than reality; they can simplify reality. There are many different types of models that we build: mental models, boxes and arrows, physical models, analytical models, computer simulation models.

The analytical models are usually solved using a spreadsheet, which uses cells to input model parameters and other output cells. It is possible to use adds-on for optimisation, inclusive of Monte Carlo simulations. However, many problems cannot be solved analytically, as a solution does not exist. In this case, there are specific characteristics: non - linear behaviour, "memory", non - intuitive influences between variables, time and casual dependencies, high uncertainty, a large number of variables. Not to mention that it is impossible to derive a mental model.

There is a dynamic dependency between variables for this class of problems and could be described as systems with dynamic behaviour, which are solved by simulation modelling.

\section{Simulation modelling}

The simulation model considers a set of rules that specifies how to get to the following systems' state from the current state, and it is always an executable model. Rules are expressed in various forms, from process flowcharts to differential equations, and is usually performed using special software - based on simulation-specific programming languages.

There are six advantages of simulation modelling: analyse systems where other methods fail, given an appropriate level of abstraction, the development of simulation model is straightforward, its structure reflects the real system, track any object/ value within the level of abstraction, play and animate the system behaviour, more convincing than classical spreadsheets.

Some applications of simulation are presented in Figure 1 [1].

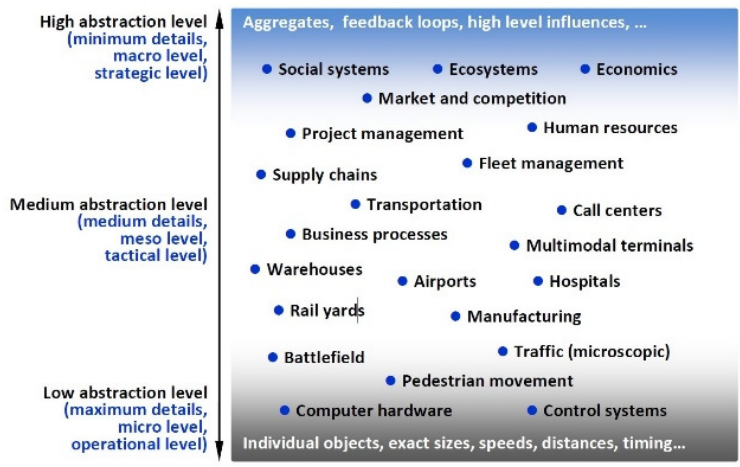

Fig. 1. Examples of areas where simulation is applied with abstraction level [1]. 
The models at the top are highly abstract, whereas, at the bottom, they have a very low level of abstraction. Ecosystems and Environmental systems are characterised by a high abstraction level, where aggregates replace individual objects.

Selecting the right abstraction level is paramount for the simulation modelling. Then, the selection of the modelling method is relatively straightforward. An indication of the suggested methods for modelling is represented in Figure 2 [1].
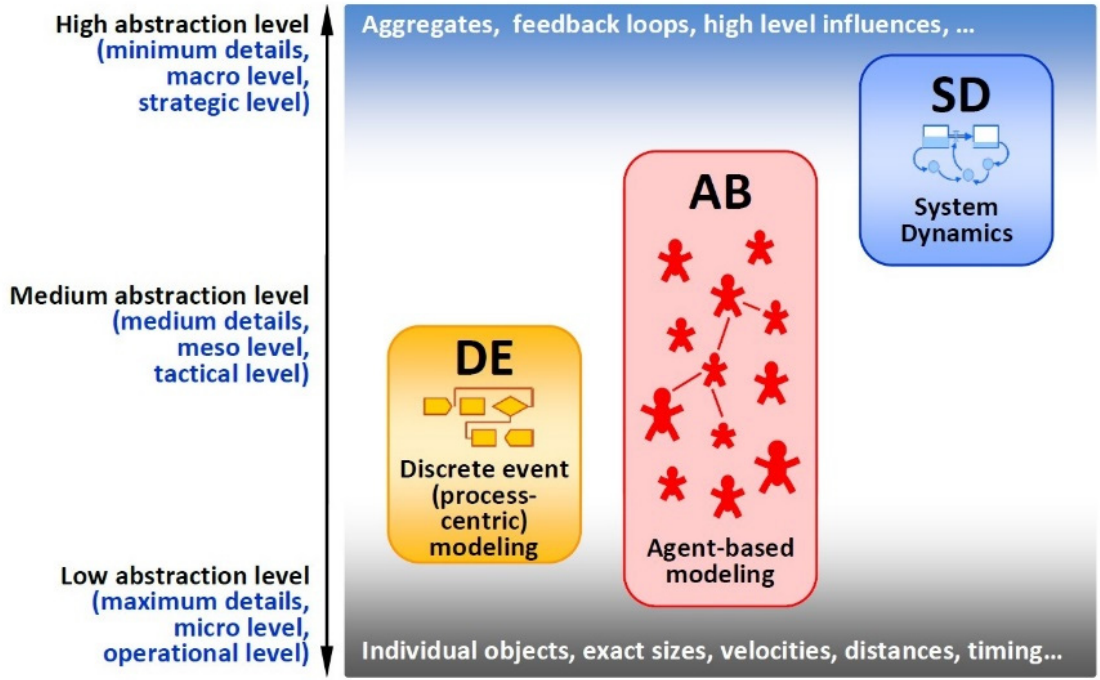

Fig. 2. Simulation methods based on abstraction level [1].

As shown in Figure 2, there are three simulation modelling methods, each serving a class of specific problems. System Dynamics (SD) is mainly used for strategic modelling, whereas Discrete Event (DE) modelling is used for a medium and low-abstraction class of problems. Agent-Based (AB) models have a more comprehensive range of applications. It is also possible to mix the modelling mentioned above Hybrid Simulation/ Modelling or Multi-Method Modelling [2].

\subsection{Systems dynamics}

System Dynamics was initially developed by Jay Forrester at MIT in the mid-1950s and studied dynamic systems. There are few characteristics: the system is modelled as a closed structure, feedback loops, stocks, and flows are the principal elements of a model.

Vensim, STELLA and AnyLogic are some of the most used software programs for Systems dynamics modelling.

\subsection{Discrete events}

In 1961, General Purpose Simulation System (GPSS) was introduced into the market by Geoffrey Gordon's from IBM. The basic modelling philosophy consists of the system being modelled as a process, with transactions/ agents as a sequence of operations - delays, resources, queues combined into a process flowchart. Discrete event simulation is based on discrete-time, with the model clock advancing when there is a change in the model.

There are hundreds of software tools for discrete event modelling, including AnyLogic. 


\subsection{Agent-based models}

It is a more recent simulation modelling technique and was developed to get a deeper insight into systems. The modelling procedure is based on a bottom-up approach by identifying agents and their behaviour.

Agents may or may not interact within the given environment, and the global behaviour is given by the millions of concurrent individual behaviours, including their dynamic creation and destruction.

AnyLogic is the only professional tool for agent-based modelling.

\section{System dynamics and agent-based models for environmental modelling}

Below are few examples of how system dynamics and agent-based models are used to model complex environmental systems.

\subsection{STELLA model - Mono Lake}

Model of Mono Lake is an inland sea on the eastern side of Sierra Nevada, close to the City of Los Angeles.

The model simulates the flows and accumulation of water in Mono Lake to evaluate the lake level changes with different policies on the water exported to Los Angeles.

The model is represented in Figure 3:

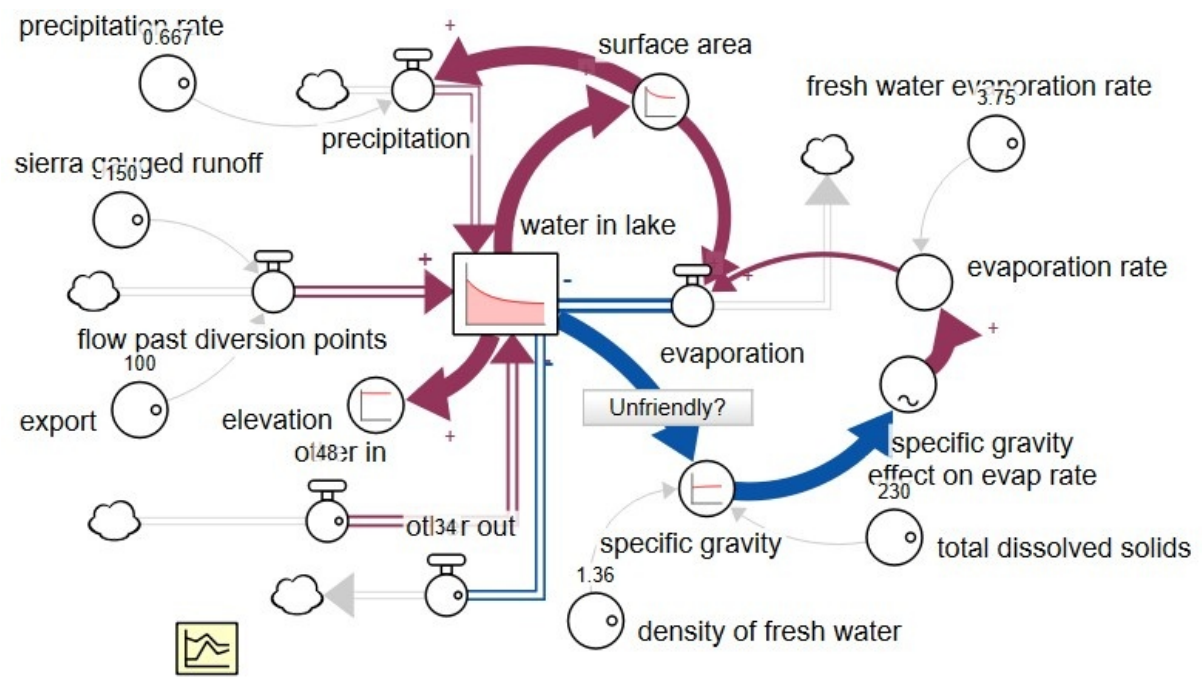

Fig. 3. Mono Lake model.

The following variables are taken into account on the current model: surface area (B1), precipitation (R1) and evaporation rate (B2).

If running the model for 100 years, the following results are derived in Figure 4. 


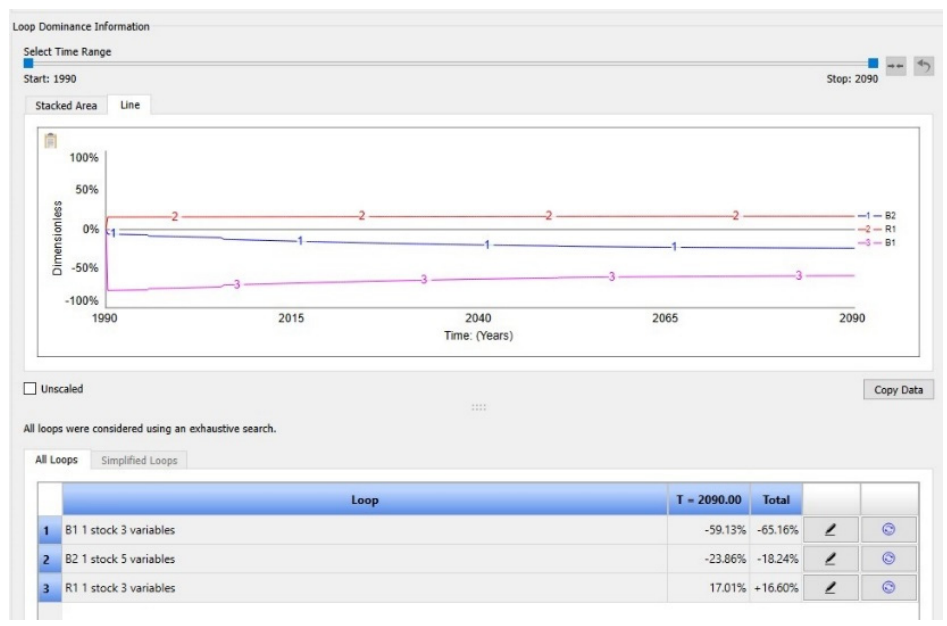

Fig. 4. Mono Lake modelling results.

\subsection{STELLA model - DaisyWorld}

Prof. Andrew Ford in "Modelling the Environment" [3] developed the model of DaisyWorld - a planet inhabited by only two plants: white daisies - having a high albedo and black daisies, with a low albedo.

The surface covered with white daisies would reflect much of the incoming solar luminosity. The black daisy covered areas would absorb this luminosity.

The mix of daisies on the planet eventually will control the temperature, which influences the rate of growth.

When the temperature is near the optimum value, there is flower growth. Vice-versa, the flowered areas will diminish in time. 6.

The simulation model is represented in Figure 5, whereas the specific B1 loop in Figure

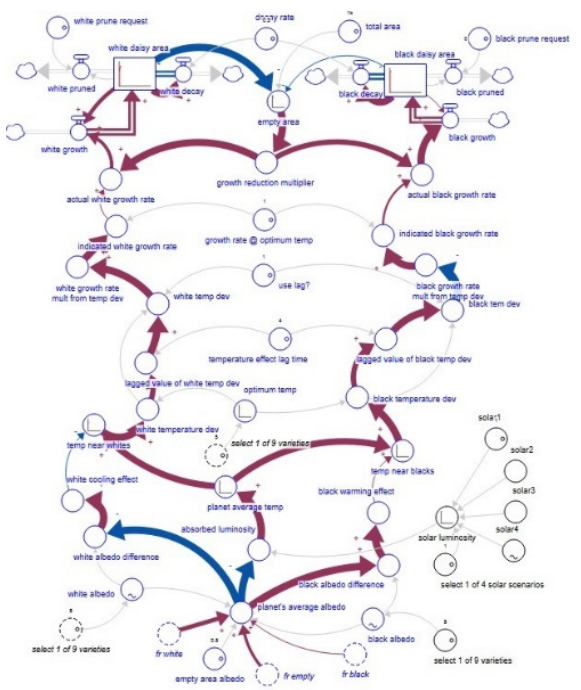

Fig. 5. DaisyWorld simulation model. 


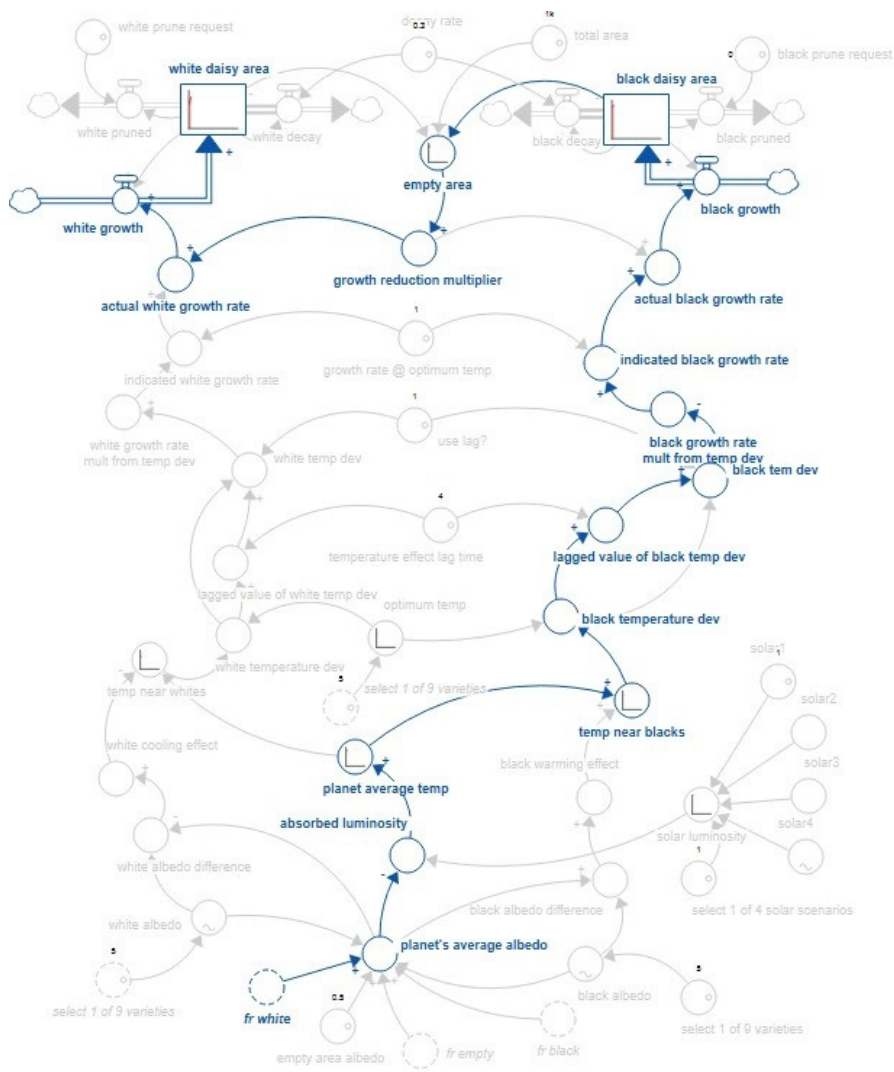

Fig. 6. Loop B1 within DaisyWorld model.

Figure 7 presents some results for three sets of loops: B1 - having three stocks and 21 variables related to black daisies, R1 - having three stocks and 21 variables related to white daisies and R2 - having three stocks and 21 variables.

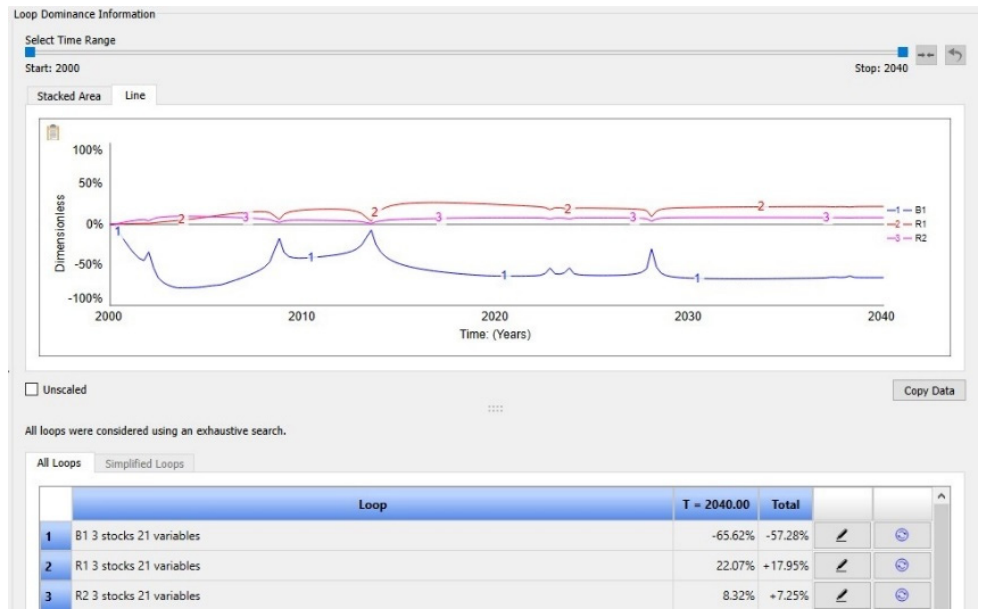

Fig. 7. DaisyWorld modelling results (first 3 loops). 


\subsection{AnyLogic model - Predator-Prey}

Figure 8 below shows a classical system dynamics model of interaction between lynx populations and hares in an isolated area. All parameters can be changed on the fly.

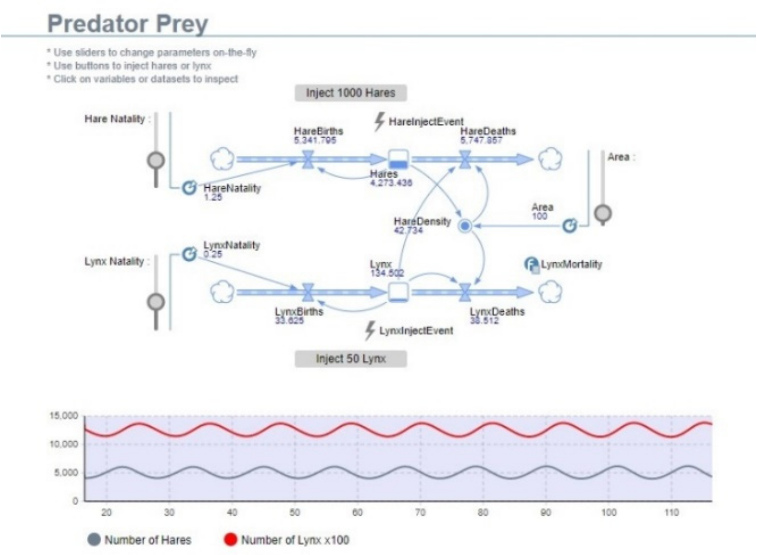

Fig. 8. Predator-Prey simulation model with results.

\subsection{AnyLogic model - wandering elephants}

An agent-based model of interaction of animals and vegetation is presented in Figure 9. Several elephants live in area 100 by 100 kilometres. There are two layers of geographical information: altitude and vegetation (we can switch between them). Elephants randomly wander in the area, but every once in a while, they get thirsty and go to the nearest lake to drink water. As elephants walk, they demolish vegetation at a specific rate. Vegetation regenerates, but it can never exceed a natural maximum that depends on the altitude.

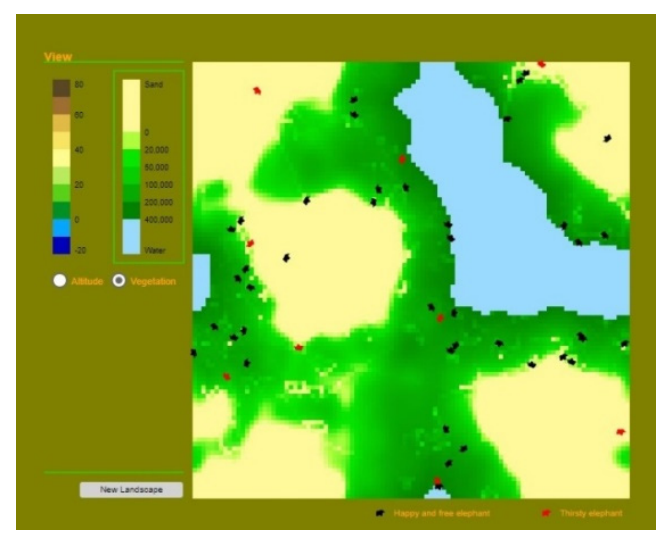

Fig. 9. Wandering Elephants model results.

\subsection{AnyLogic model - climate change}

A model to simulate global warming and climate change is shown in Figure 10. It contains all system-dynamic variables and dependencies.

The model's dashboard is depicted in Figure 11, where we can select the target period of carbon reduction and the number of years to reach the goal. 


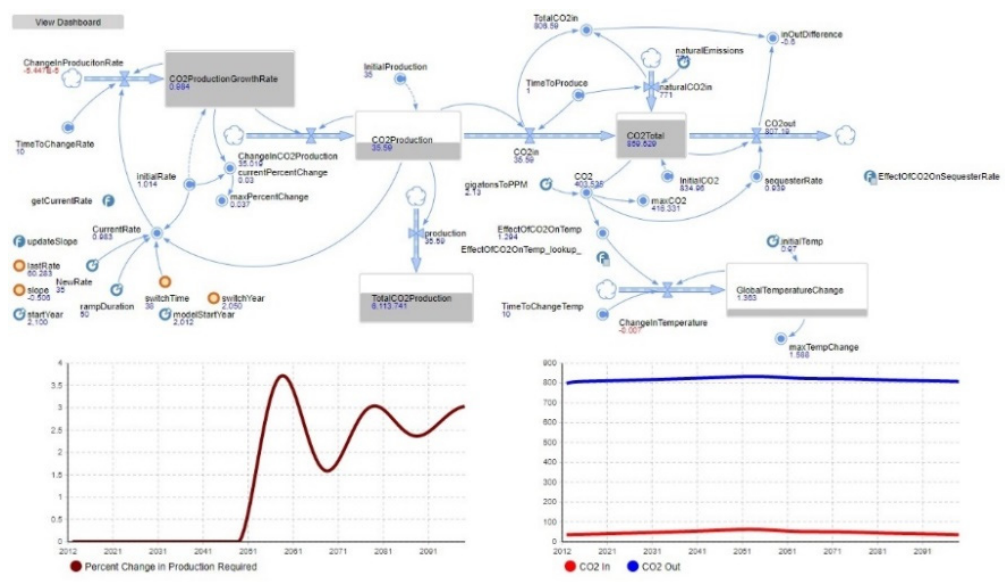

Fig. 10. Climate change model and results.
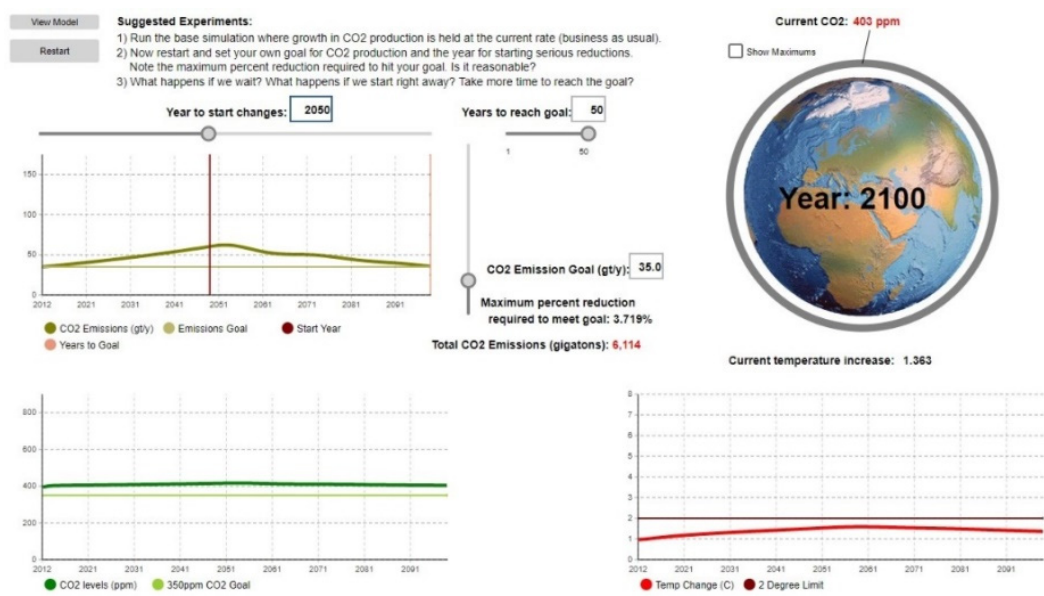

Fig. 11. Climate change dashboard.

\section{Conclusions}

Environmental modelling represents a valuable tool towards sustainability by helping us understanding the challenges if the models follow the feedback effects that interconnect the environmental, social, and economic systems into a tightly coupled system [3].

Simulation can be used in a wide range of environmental problems, including climate change problems as presented in this paper, and it is helpful in interdisciplinary modelling.

Systems Dynamics and Agent-Based Modelling and Hybrid Modelling are the most used and recommended methods for Environmental Modelling.

\section{References}

1. A. Borschev, The Big Book of Simulation Modelling. Multimethod Modeling using AnyLogic 6 (eBook)

2. A. Mahdavi, The Art of Process-Centric Modeling, with AnyLogic (eBook)

3. A. Ford, Modeling the Environment, Second Edition (Island Press, Washington, 2010) 